\title{
Design and Implementation of Distress Prevention System using a Beacon
}

\author{
Imsu Lee ${ }^{1}$, Kyeonhoon Kwak ${ }^{1}$, Jeonghyun Lee ${ }^{1}$, Sangwoong Kim ${ }^{1}$, Daehan Son ${ }^{1}$, Eunju Park ${ }^{1}$ and Hankyu Lim ${ }^{1 . a}$ \\ ${ }^{1}$ Department of Multimedia Engineering, Andong National University, Republic of Korea
}

\begin{abstract}
It was proven that human accidents due to mountain climbing occupy large proportion of recently occurred man-made disasters. This paper designed and implemented an application that tells users about a variety of accidents that frequently occur in mountain and shows dynamic information about mountain climbing. It designed 'distress prevention system using beacon'. When using beacon, it can dynamically express information without using GPS. It has advantage of supporting BLE and has little Smartphone battery consumption. The application was developed based on Android as a prototype. We expect that the application developed in this paper can contribute to the protection of precious lives by helping quick rescue in case of emergency such as distress. Manager of mountain can control data regarding the mountain climbing accident where the related functions are provided through view and user authority setup in DBMS.
\end{abstract}

\section{Introduction}

As perception about the importance of health gets stronger, the number of people interested in mountain climbing is increasing. Accordingly, mountain climbing accidents or distress in mountain is becoming more frequent. '2013 Disaster Yearbook' by the Ministry of Public Safety and Security regarding the situation of human disaster occurrence indicates very high frequency of human damage from mountain climbing, along with car accidents, falling and bicycle accidents [1]. However, national point number system that marks location information with signs is the best response to mountain climbing accidents as of now which has location limits as it has a form of sign [2]. In this situation, prompt response is almost impossible when accidents or critical situation happens. Hence, this paper attempts to provide dynamic and quick information regarding mountain climbing by using beacon to decrease accidents occurrence and help quick rescue in case of critical situation. Moreover, we believe that mountain climbing information can be made to data to be accumulated when using this application and that they can be used as other contents based on Big Data. Implementation in this paper a test application developed based on Android as a prototype.

\section{Related Application}

'Rambler' by Bientus and 'Sanddara Baramddara' by Baramgaebi are the representative domestic applications that are related to mountain climbing [3][4]. Figure 1 shows the implementation look of 'Rambler' and 'Sanddara Baramddara'.

'Rambler' is a service that marks users' record on mountain climbing activity on the map by using GPS[5]. Users can obtain information about mountain trail through map and can also see information about mountain trail that other users recorded alongside media. The recorded information can be used as diverse statistics data and they are linked to web service.

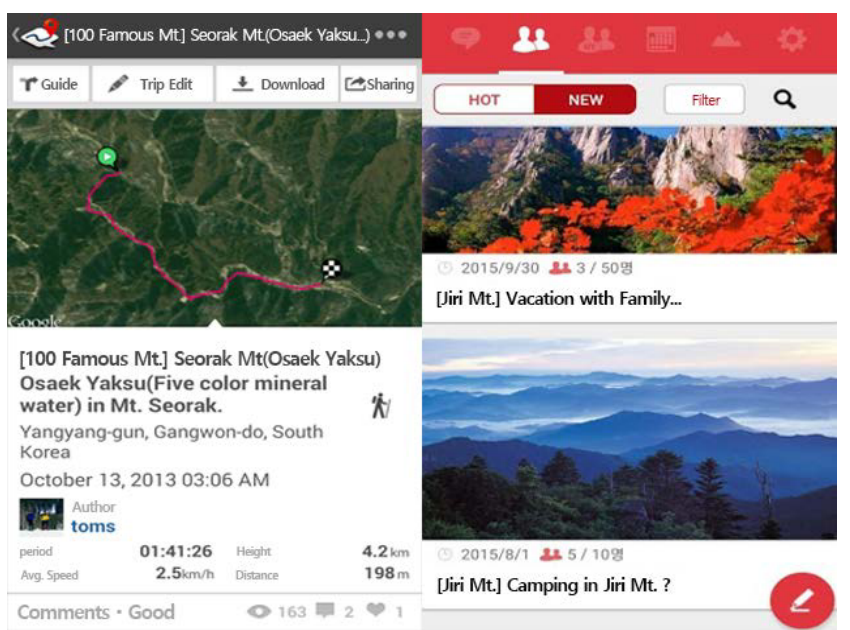

Figure 1. The Famous Application of Mt. Climbing

'Sanddara Baramddara' is an application that provides information about nationwide mountains, similar to most of the mountain-related applications. Without special function, it helps users search for desired mountains easily and supports communication such as comment.

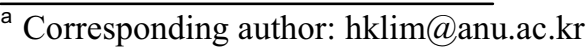


In this paper, basic information about domestic mountains is provided and records on mountain climbing activity are implemented. As a major function, mountain climbing information is shown to users in real time through beacon.

\section{Major Function and Restriction Element}

Major function of the system implemented in this paper contains the following. First is information notification service through beacon. Second is record on mountain climbing activity. Third is record on distress.

Figure 2 shows the principles of service function of beacon [6].

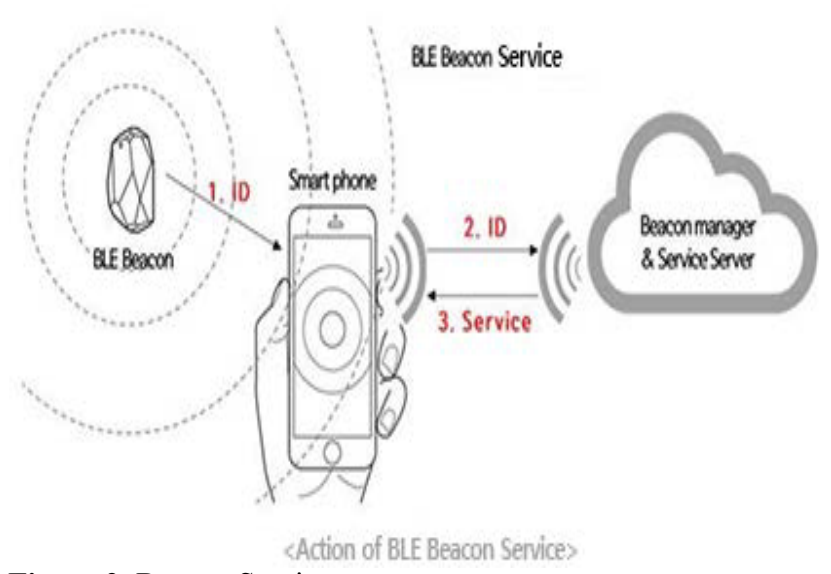

Figure 2. Beacon Service

Beacon is a location-based communication technology that attempts Bluetooth communication in one direction to user application SDK as shown in Figure 2 [7]. Beacon is a simple device that delivers only id values to users. Id value is identified in SDK and communication with server is made by using this value as a factor.

The role of beacon is to prepare a chance for communication with server when user enters into a relevant range. Here, the relevant range is the Bluetooth communication distance, which is up to $70 \mathrm{~m}$. In this paper, beacon signal captured by Bluetooth function while mountain climbing is analyzed to extract id values and the corresponding data are retrieved from DB to provide adequate application table and push message.

The second major function is record on the mountain climbing activity. The application implemented in this paper shows information about mountain, date and time of mountain climbing and passage.

Finally, the most important function, that is, the function in case of distress is as follows. Beacon is installed in locations that is not mountain trail or is a zone susceptible to distress and warning is given when user enters this point. Here, the previously stored GPS coordinate of beacon is expressed on map to indicate the user's current location and direction for the route back to mountain trail is provided along with a compass. As this function is map information that is provided with the stored data, no GPS sensor is necessary. In case of injury or when call is required, user can easily contact management office with the provided calling connection function. If communication is not possible, call can be made through server by using call button. Moreover, if there is no movement at the spot for a certain period of time or if one crosses the distress point more than twice, it is regarded as emergency situation and user information and location information are transferred to the server. It is expected that management office can easily recognize the information and promptly respond to the situation. To use this kind of function, users should always turn on Bluetooth while mountain climbing. The current BLE beacon has a feature of little battery consumption of Smart mobile devices compared to previous Bluetooth protocols [8]. Table 1 below presents the limiting factors in the process of system design in this paper.

Table 1. Restriction Element

\begin{tabular}{|c|c|l|}
\hline \multicolumn{2}{|c|}{ Restriction Element } & \multicolumn{1}{|c|}{ Reflection Contents } \\
\hline \multirow{2}{*}{ Theme } & Social & Development for wide use \\
\cline { 2 - 3 } & Economy & $\begin{array}{l}\text { Minimizing development cost with } \\
\text { Android open source }\end{array}$ \\
\hline \multirow{4}{*}{ External } & Ethics & Information based on Ethics \\
\cline { 2 - 3 } & Beauty & $\begin{array}{l}\text { Considering UI, UX based on } \\
\text { beauty }\end{array}$ \\
\hline \multirow{3}{*}{ SW } & Security & $\begin{array}{l}\text { Minimizing and securing private } \\
\text { information }\end{array}$ \\
\cline { 2 - 3 } & Productivity & iOS 7.0, Android 4.3, Bluetooth \\
\cline { 2 - 3 } & Standard & Observing mobile app. standard \\
\hline
\end{tabular}

\section{Design}

\subsection{Data Flow Diagram (DFD)}

Figure 3 and Figure 4 below shows the schematization of system data flow.

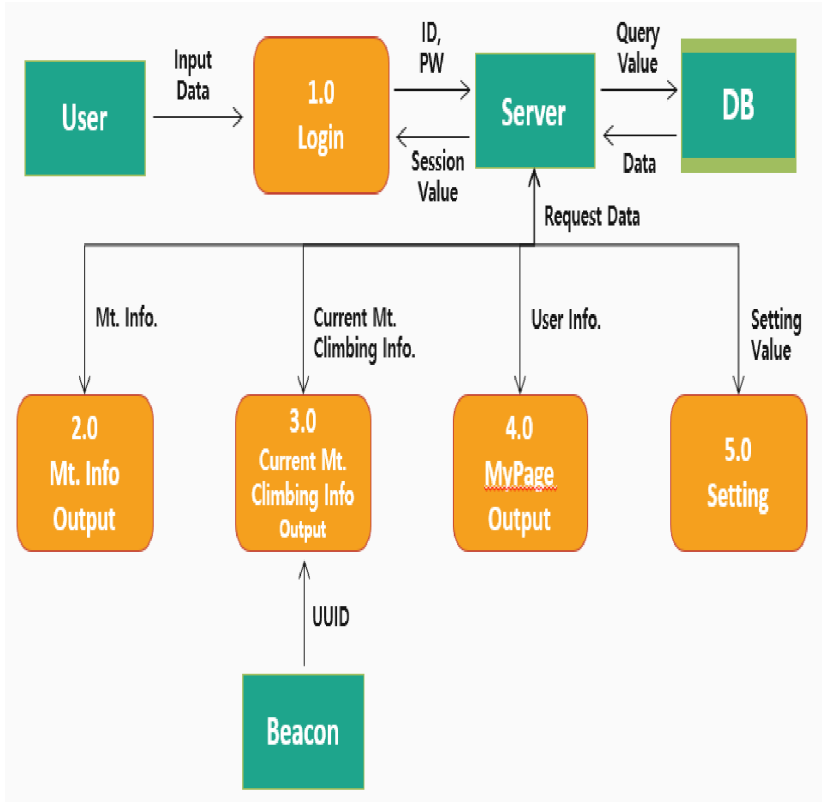

Figure 3. DFD scheme 


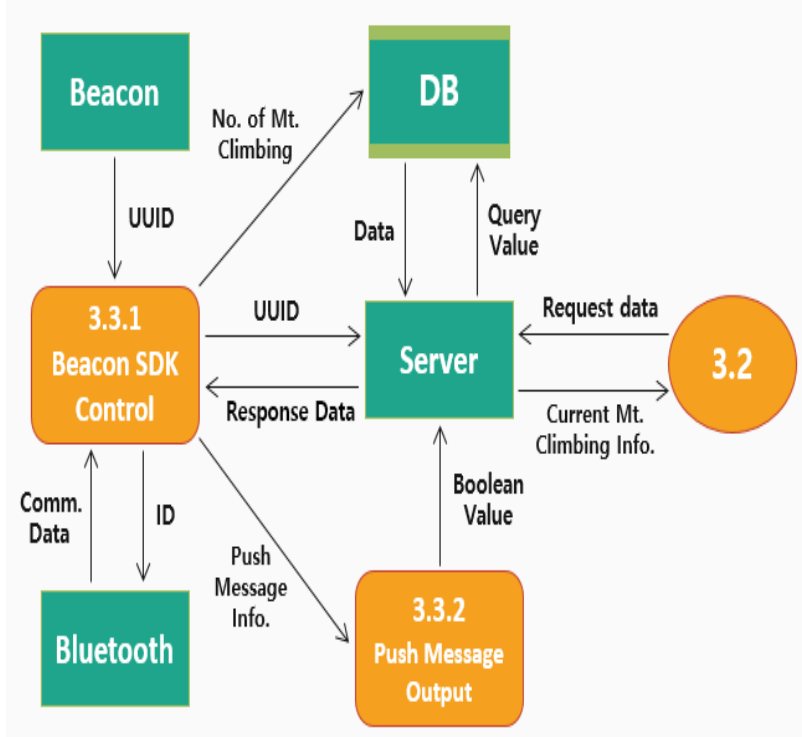

Figure 4. Beacon process DFD

\subsection{Flow Chart and Scenario}

Figure 5 below shows the overall flow chart of this system.

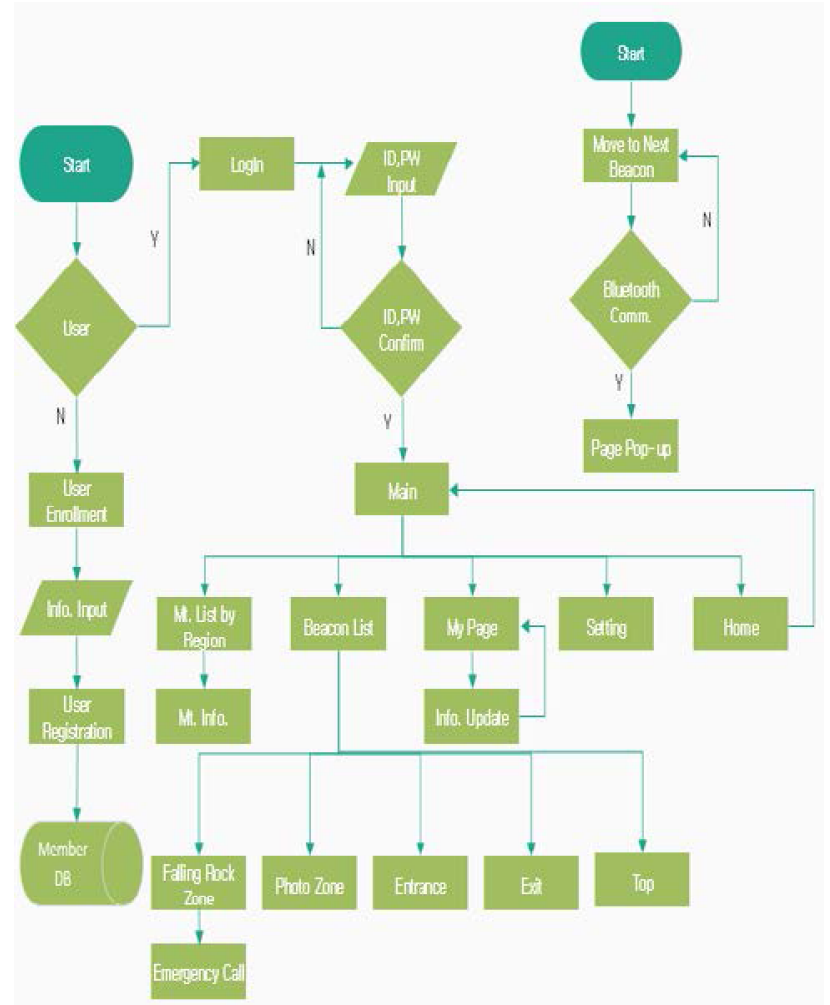

Figure 5. Flowchart

First, users apply for membership with simple personal information that can be helpful in case of distress and log on. The log-in information continuously maintains session in local domain. Afterward, beacon function is turned on at entrance and entrance beacon is recognized with an 'entrance finding' button. From here, beacons in the relevant mountain can be recognized and information is provided. It is designed such that Bluetooth is automatically turned on and turned off once mountain climbing finishes.

Next, there are six types of beacon that is recognized in case of mountain climbing, which include 'peak', 'photo zone', 'hazard zone', 'distress point', 'exit' and 'entrance'. 'Entrance' provides the relevant entrance information and begins counting the mountain climbing time. 'Peak' provides relevant peak information and notifies peak

arrival time. 'Photo zone' provides information about the surrounding landscape with image and recommends spots for taking pictures. 'Hazard zone' tells risk of relevant location such as frozen road, falling rock zone and steep slope to prevent accidents in advance. As was mentioned earlier, 'distress point' provides map information along with compass and has a calling function. Finally, when the user arrives at exit, 'exit' beacon is perceived to store the record on the mountain climbing activity so far and lists them to display. A push message is also serviced which connects to a relevant table once a beacon is perceived. Even when user climbs mountain while shutting the Smartphone screen off, he/she can dynamically receive mountain climbing information from the push message.

\subsection{Entity Relationship Diagram (ERD)}

The application in this paper consists of a total of nine tables whose relationship is depicted in Figure 6.

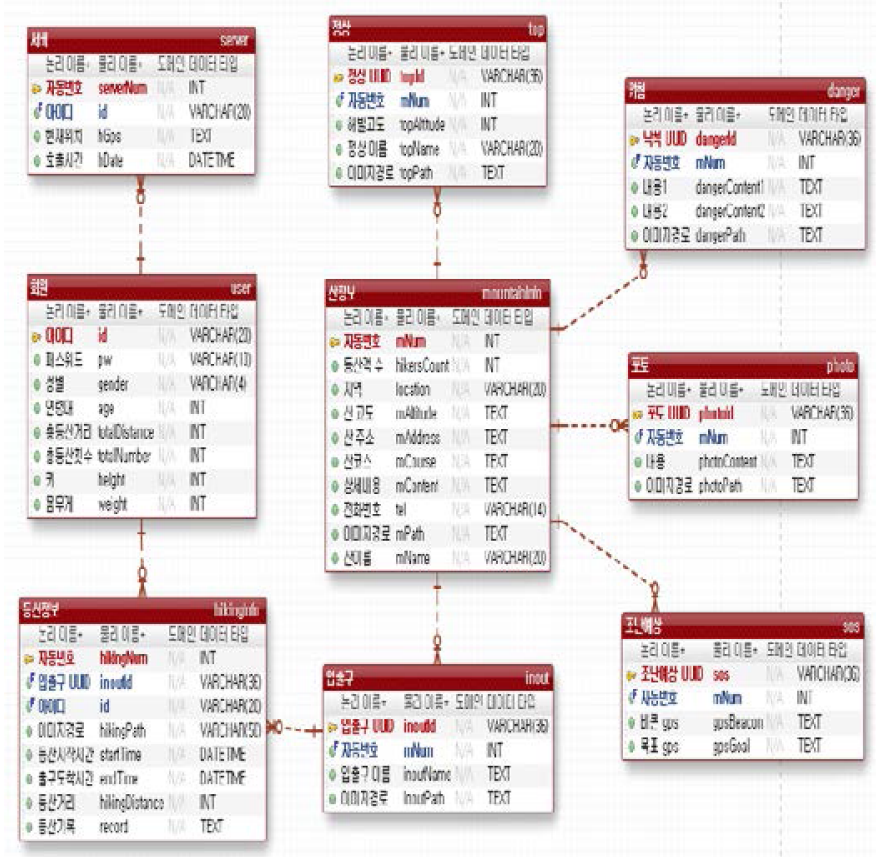

Figure 6. ERD

\section{Implementation}

'Distress prevention system using beacon' application was implemented by using java-based Android programming. Figure 7, Figure 8 and Figure 9 below show the actual screen implemented in Smartphone. 


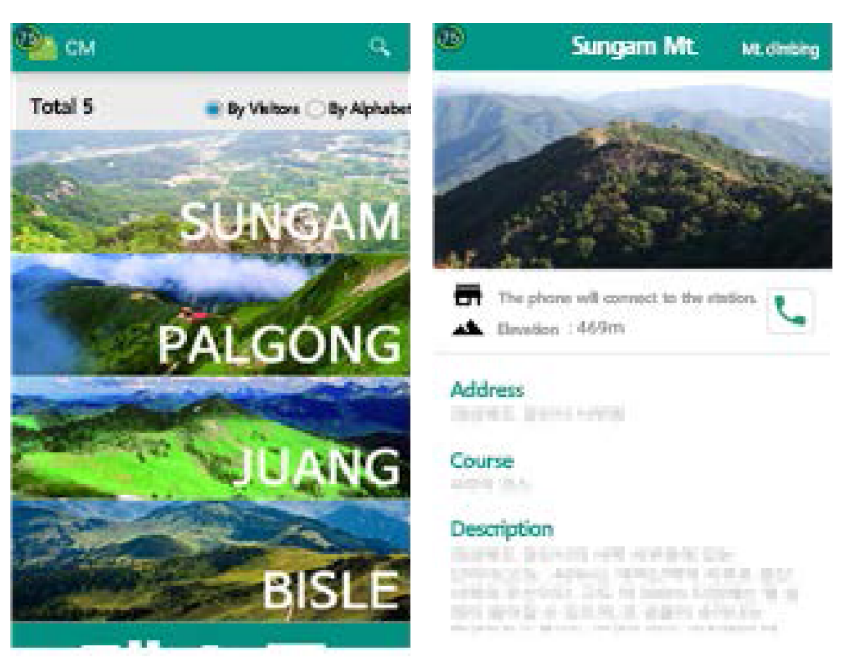

Figure 7. Mt. List and Mt. Information

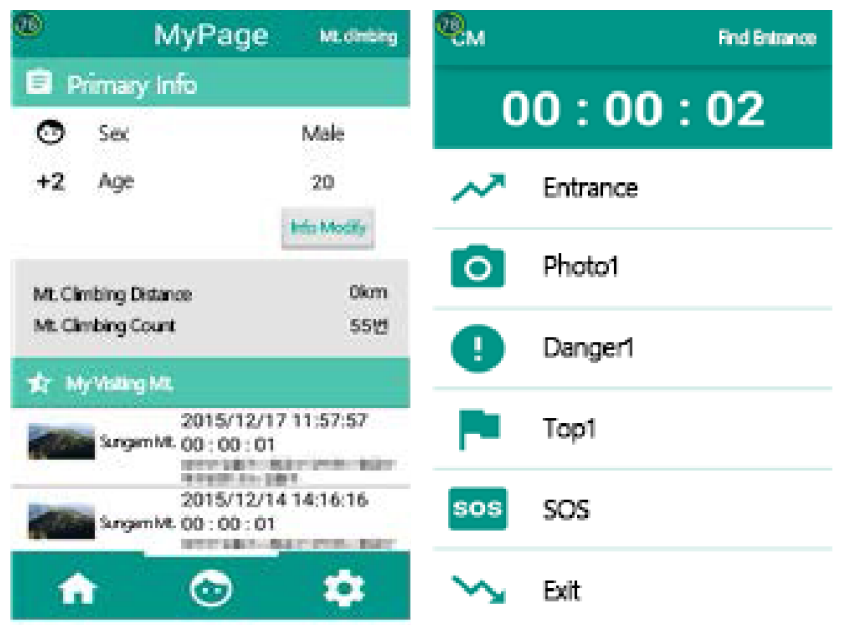

Figure 8. User Info. and Beacon Info. List

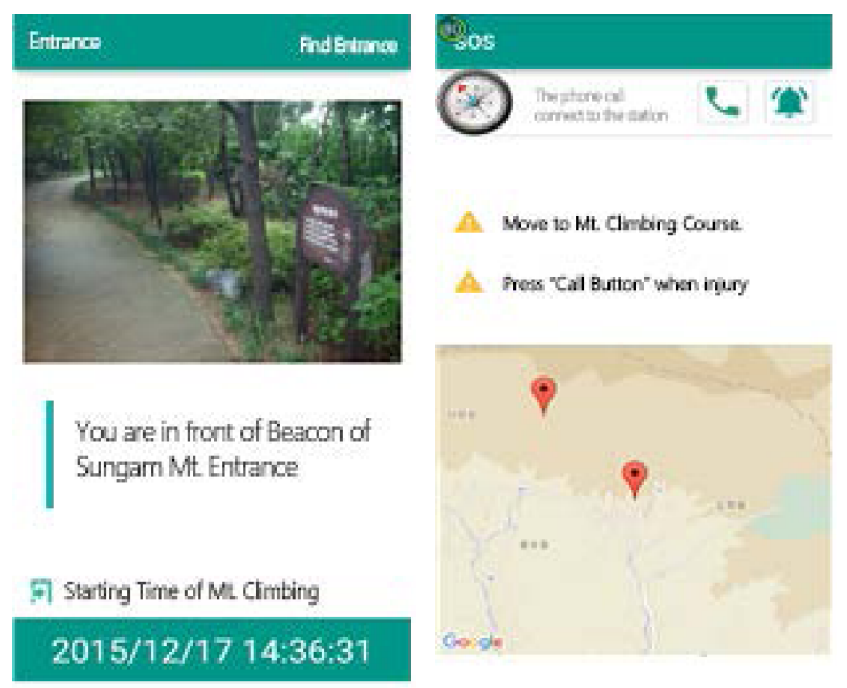

Figure 9. Activated Page with Beacon

Figure 10 shows the captured screen of implementation source of beacon scan function of the application. The function captures beacon signal by using Bluetooth library of Android and returns requested data.

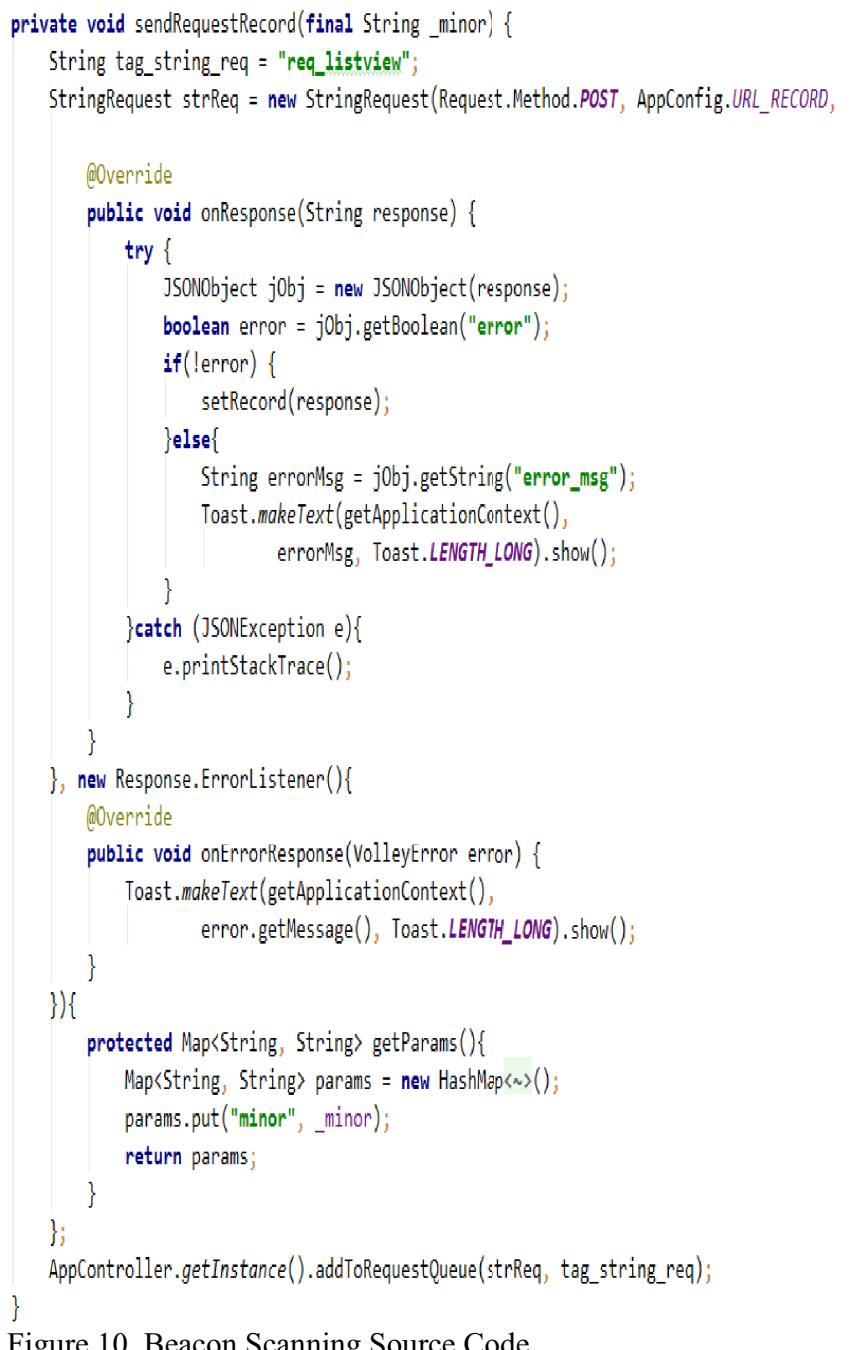

This system provides data on mountain climbing accident through view and user authority setup in DBMS. Figure 11 shows the implementation of 'user view' and 'mountain climbing information view' of DBMS. View table is created to provide only the information that can be given to administrator.

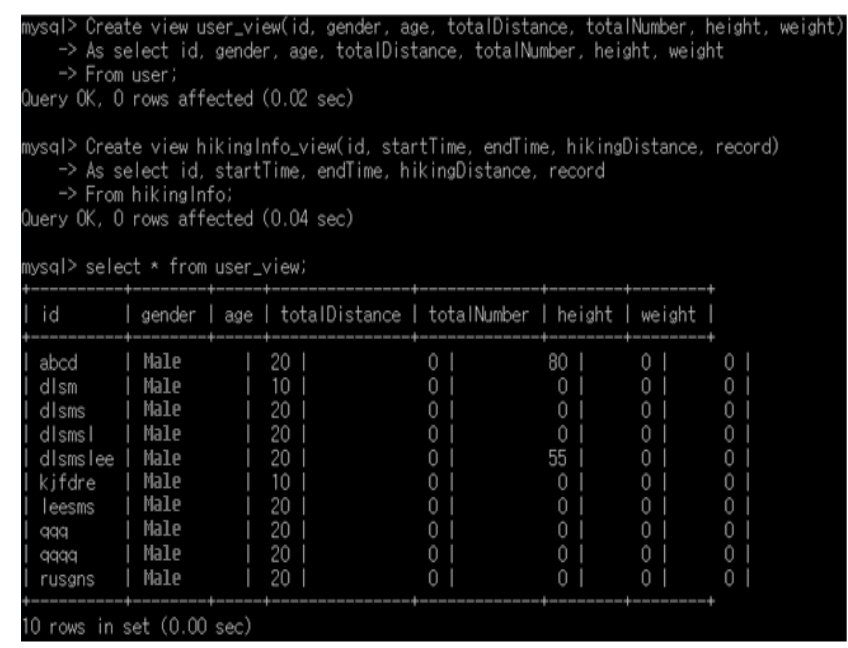

Figure 11. 'User View' and 'Mt. Climbing View'

Figure 12 implements the authority given to administrator so that administrator can directly use the information coming to web server table in a limited manner. 


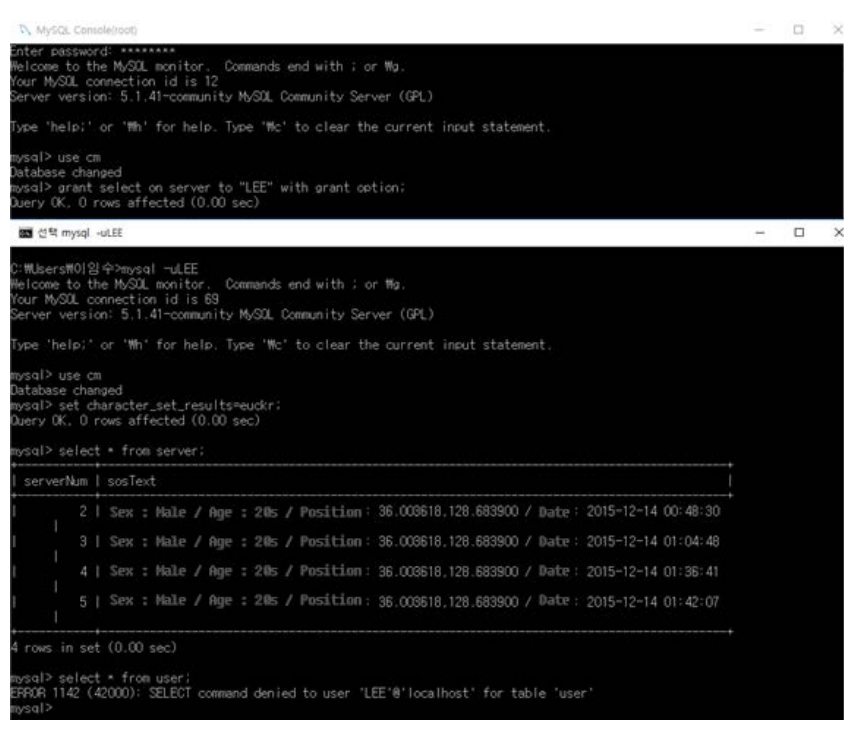

Figure 12. Managing Authority with 'LEE'

\section{Conclusion}

This paper designed 'distress prevention system using beacon' and implemented it in a form of application. When using beacon, it can dynamically express information without using GPS. Moreover, it has advantage of supporting BLE (Bluetooth Low Energy) and has little Smartphone battery consumption. Moreover, manager of mountain can control data regarding the mountain climbing accident where the related functions are provided through view and user authority setup in DBMS.

Using the system implemented in this paper, developing contents based on Big Data is also possible by accumulating information about when and how a certain age group went to mountain climbing. Moreover, it is expected to grow to be ICT convergence contents that can replace the national point number system.

Acknowledgments: This work was supported by a grant from 2016 Seoul Accord Project (R0613-16-1148) of MISP (Ministry of Science, ICT and Future Planning) and IITP (Institute for Information and Communication Technology Promotion).

\section{References}

1. Ministry of Public Safety and Security, "2013 Disaster Yearbook-Human Disaster", Ministry of Public Safety and Security (2013)

2. Ministry of the Interior Blog, http://mogahablog. net/11811027

3. Bientus' Rambler, http://www.rambler.com/web/ma in

4. Baramggabi's Sanddaramulddara, https://play.google.com/store/apps/details?id=windm ill.windmill\&hl=ko

5. K. Kwak, M. Yoon, Y. Lim, H. Lim, “A Design and Implementation of Vertical SNS for Student Backpackers", Proceedings of the International
Conference on Communications and Computers, Oct. 2015, pp. 11 - 13

6. Beacon Principle, http://blog.lgcns.com/565

7. S. B. Han, J. H. Song, "Product Information Push Technique using Beacon MacAddress", Journal of The Society of Convergence Knowledge, Vol. 4, No. 1 (2016)

8. H. Namgung, "A System of Measurement Signal Analysis and Comparison using Bluetooth Low Energy Beacon", Graduate School of Hannam University (2015) 